\title{
An observation study of the prognostic effect of waiting times in the management of pancreatic ductal adenocarcinoma
}

\author{
REI SUZUKI ${ }^{1}$, TADAYUKI TAKAGI ${ }^{1}$, TAKUTO HIKICHI ${ }^{2}$, MITSURU SUGIMOTO ${ }^{1}$, NAOKI KONNO ${ }^{1}$, \\ HIROYUKI ASAMA $^{1}$, KO WATANABE ${ }^{1}$, JUN NAKAMURA ${ }^{2}$, SHIGERU MARUBASHI $^{3}$ and HIROMASA OHIRA ${ }^{1}$ \\ ${ }^{1}$ Department of Gastroenterology, Fukushima Medical University School of Medicine; ${ }^{2}$ Department of Endoscopy, \\ Fukushima Medical University Hospital; ${ }^{3}$ Department of Hepato-Biliary-Pancreatic and Transplant Surgery, \\ Fukushima Medical University School of Medicine, Fukushima 960-1295, Japan
}

Received March 30, 2018; Accepted September 12, 2018

DOI: $10.3892 / \mathrm{ol} .2018 .9626$

\begin{abstract}
Pancreatic ductal adenocarcinoma(PDAC) is characterized by an aggressive course; therefore, it was hypothesized that waiting times during disease management may serve as a prognostic factor for patients with PDAC. Data for all patients with PDAC who received treatment in Fukushima Medical University Hospital were collected. Median disease-free survival and overall survival time were calculated using the Kaplan-Meier method and utilized as cut-off points to divide the patients into 2 groups: A short and a long survival group. Clinical characteristics, including waiting times, the detection-to-diagnosis waiting time and the diagnosis-to-treatment waiting time, were compared between the 2 survival groups. A total of 149 patients were included in the present study. Among the 72 patients who underwent chemotherapy, no significant differences between the 2 survival groups regarding waiting times were identified; however, the proportion of patients with locally advanced disease and the administration of combination chemotherapy were significantly associated with increased survival. Additionally, no significant differences in the waiting times between the 2 survival groups were identified when evaluating the 79 patients who underwent surgical resection. In conclusion, the results of the present study indicated that detection-to-diagnosis and diagnosis-to-treatment waiting times do not influence the prognosis of patients with PDAC.
\end{abstract}

\section{Introduction}

Pancreatic ductal adenocarcinoma (PDAC) is one of the most fatal malignancies according to cancer statistics of USA in 2016 (1). Surgical resection, to the best of our knowledge,

Correspondence to: Dr Rei Suzuki, Department of Gastroenterology, Fukushima Medical University School of Medicine, 1 Hikarigaoka, Fukushima 960-1295, Japan

E-mail: subaru@fmu.ac.jp

Key words: pancreatic ductal adenocarcinoma, waiting time, prognosis, chemotherapy, surgery is currently the only curative treatment for PDAC; however, $<20 \%$ of patients are potential candidates for pancreatectomy as the majority are diagnosed at the advanced disease stage (2). Furthermore, even following successful surgical resection, it has been reported that the 5-year survival rate following pancreatectomy is only $10-20 \%$ due to a high rate of disease recurrence according to a German study conducted between 1998-2003 (3), a Korean study conducted between 1983-2011 (4) and a USA study conducted between 2003-2010 (5). Recently established chemotherapy regimens, including folinic acid, fluorouracil, irinotecan and oxaliplatin (FOLFIRINOX) and gemcitabine plus nab-paclitaxel therapy, have improved the prognosis of patients with PDAC (6-9). However, the median survival time among patients who underwent the aforementioned chemotherapy regimens has been reported as $<1$ year according to literatures published as international collaborative studies, which included patients from 11 countries and Japanese domestic studies conducted between 2003-2014 (6-9). Given the challenges associated with altering the biological behavior of PDAC, it is important to continue investigating additional ways to improve patient prognosis.

The length of waiting until medical care has drawn increasing attention in previous studies as a potential factor that may affect the prognosis of patients with various types of malignancies (10-16). Based on previous studies, waiting times can be classified into four categories: i) Waiting time until the first medical visit; ii) waiting time until detection of the disease; iii) waiting time until diagnosis; and iv) waiting time until treatment (10-16). The impact of waiting time on patients' prognosis varied in each study, but a number of the patients revealed reduced outcome with long waiting time, compared with patients with a reduced waiting time $(13,15)$. Although a literature search identified 3 reports in which the authors investigated the prognostic effect of waiting time on the survival of patients with PDAC, none of the previous studies, to the best of our knowledge, evaluated the effect of waiting time between the detection of disease and diagnosis (11-13). Endoscopic ultrasound-guided fine-needle aspiration (EUS-FNA) has been accepted globally as the gold standard for diagnosing PDAC and may be used to obtain highly accurate diagnoses $(17,18)$. However, in contrast to the diagnostic yields of other modalities, 
the diagnostic yield of EUS-FNA is highly dependent on the expertise of the clinicians, cytoscreeners and the pathologists performing this procedure and assessing the results (19-21). Consequently, patients who require EUS-FNA are referred to a limited number of tertiary referral facilities and are have to wait for a certain length of time prior to undergoing EUS-FNA to obtain a pathological diagnosis. For example, patients had to wait for 45.2 days until they underwent their second EUS-FNA following the initial EUS-FNA (20). Additionally, patients are required to wait prior to commencing treatment, which may be due to the high demand for pancreatic specialists $(11,12)$. The aim of the present study was to clarify whether the length of the waiting times to diagnosis and treatment affected the prognosis of patients with PDAC.

\section{Materials and methods}

Patient sample collection. A retrospective observational study was conducted to review the data obtained from patients histologically diagnosed with PDAC using EUS-FNA, who were treated at Fukushima Medical University Hospital (Fukushima, Japan) between January 2006 and July 2016. A total of 149 patients were included in the present study (mean age, 67.2 years; age range, 42.0-86.0; 83 males and 66 females). All patients who underwent scheduled chemotherapy or surgical resection with known final outcomes were included. To eliminate the possibility of selection bias in treatment, the present study included only the patients who were able to undergo the treatment that was originally recommended at the time of diagnosis. In the case of a patient who was originally scheduled to undergo surgical resection, but tumor cell dissemination was subsequently observed during the surgical laparotomy, the patient was excluded from the analysis, as it was not clear whether the prolonged waiting time was the cause of the tumor cell dissemination or if it was originally present as occult metastasis. In cases involving chemotherapy, first-line chemotherapy regimens that included monotherapy (gemcitabine or S-1) and combination therapy (FOLFIRINOX or gemcitabine plus nab-paclitaxel) were selected based on the Eastern Cooperative Oncology Group Performance Status Scale (22) and comorbidity of the patients. Due to the national health insurance coverage, monotherapy was selected as the first-line chemotherapy between April 2006 and December 2013. FOLFIRINOX was available in December 2013 and gemcitabine plus nab-paclitaxel was added to the list in December 2014. Second-line chemotherapy regimens were selected based on the physician's discretion, taking into consideration the patients' general condition. In cases involving surgical resection, all the patients underwent surgical resection with curative intent, and those who received preoperative chemoradiation therapy were excluded from the present study. Patients were followed up using imaging modalities, including computed tomography, every 2-3 months following the initiation of the primary treatment. Median disease-free survival (DFS) and overall survival (OS) time following surgical resection were calculated using the Kaplan-Meier method and were each utilized to determine the cut-off points to divide patients into 2 groups: A short and a long survival group, with the median value included within the long survival group. For further
Table I. Clinical characteristics of all patients.

\begin{tabular}{lc}
\hline Chemotherapy $(\mathrm{n}=72)$ & \\
\hline Characteristics & Median (range) \\
\hline Age (years) & $66.0(59.0-68.0)$ \\
Tumour size (longest diameter; mm) & $35.0(25.0-47.5)$ \\
CEA (ng/ml) & $4.2(2.4-9.5)$ \\
CA19-9 (U/ml) & $1,196.0(157.4-4,899.0)$ \\
WT1 & $20.0(11.0-28.0)$ \\
WT2 & $17.5(9.0-29.0)$ \\
Sex & 39 \\
Male & 33 \\
Female & 37 \\
Tumour location & 35 \\
Ph & \\
Pbt & \\
Disease stage (UICC ver.7) (24) & 53 \\
Locally advanced & 50 \\
Metastatic & \\
Chemotherapy & \\
Monotherapy & \\
Combination & \\
Su & \\
\hline
\end{tabular}

Surgery $(n=77)$

Characteristics

Median (range)

Age (years)

$71.0(64.0-76.0)$

Tumour size (longest diameter; $\mathrm{mm}) \quad 19.0$ (15.0-25.0)

CEA (ng/ml)

$2.8(1.9-6.0)$

CA19-9 (U/ml)

$102.8(25.8-333.5)$

WT1

$21.0(14.0-36.0)$

WT2

$46.0(29.0-60.0)$

\section{Sex (n)}

Male

Female

Tumour location (n)

$\mathrm{Ph}$

$\mathrm{Pbt}$

UICC T stage (n) (24)

T0-1

10

T2

12

T3-T4

55

UICC N Stage (n)

No

49

N1

28

Adjuvant chemotherapy

Yes

48

No

CEA, carcinoembryonic antigen; CA19-9, cancer antigen 19-9; WT1, detection-to-diagnosis waiting time; WT2, diagnosis-to-treatment waiting time; $\mathrm{Ph} / \mathrm{b} / \mathrm{t}$, pancreatic head/body/tail; T, tumor; $\mathrm{N}$, node; UICC, Union for International Cancer Control classification. 
Table II. Comparisons of waiting time between patients underwent surgery $(n=79)$ and cancelled surgery $(n=5)$.

\begin{tabular}{lccc}
\hline Characteristics & Surgery $(\mathrm{n}=79)$ & Cancelled surgery $(\mathrm{n}=5)$ & P-value \\
\hline WT1, days (range) & $21.0(14.0-36.0)$ & $20.0(15.0-30.0)$ & 0.70 \\
WT2, days (range) & $46.0(29.0-60.0)$ & $43.0(30.0-55.0)$ & 0.75 \\
\hline
\end{tabular}

Mann-Whitney U-test was applied for comparison of continuous variables. WT1, detection-to-diagnosis waiting time; WT2, diagnosis-to-treatment waiting time.
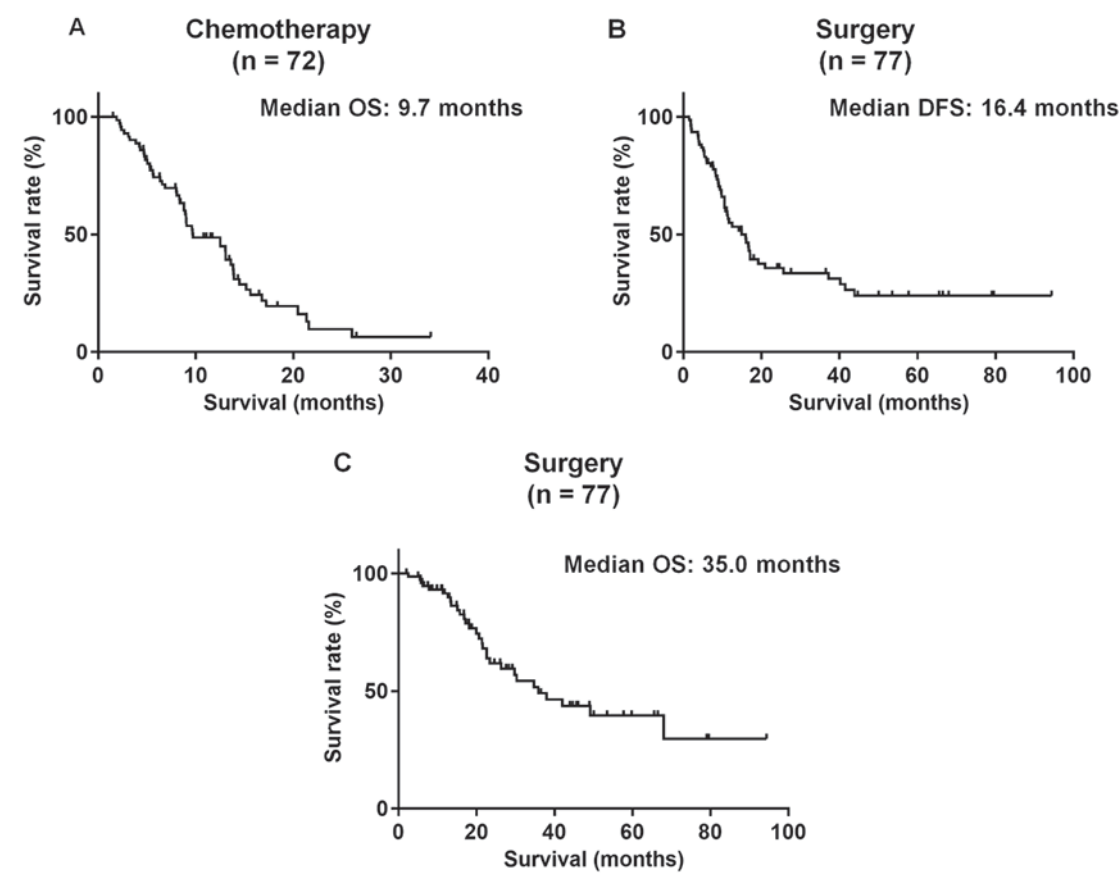

Figure 1. Survival analysis of patients who underwent chemotherapy and surgical resection. (A) The median OS was 9.7 months for patients who received chemotherapy. In the surgical resection group, the median (B) DFS was 16.4 months, and the median (C) OS was 35.0 months. DFS, disease-free survival; OS, overall survival.

analysis, clinical data on patients in the lower and upper quartiles with respect to detection-to-diagnosis waiting time (WT1) and diagnosis-to-treatment waiting time (WT2) were obtained in order to assess the prognostic effect of immediate and delayed treatment. Survival analysis was subsequently performed using the Kaplan-Meier method with log-rank tests to compare the two groups for each type of treatment.

Variables. Waiting times to diagnosis and treatment were estimated to be the time from the detection of disease with imaging (computed tomography) to histological diagnosis using EUS-FNA (WT1), and the time from histological diagnosis to treatment initiation (WT2). OS was defined as the time from treatment initiation to mortality from any cause. In cases involving surgery, DFS was defined as the survival period during which patients survived with no signs of recurrence following surgical resection. Clinical characteristics prior to the initiation of primary treatment, including age, sex, tumor size, tumor location, Tumor ( $\mathrm{T}$ ) and Node (N) stages based on the Union for International Cancer Control classification, ver. 7 (23), serum levels of tumor markers, including carcinoembryonic antigen and cancer antigen 19-9, waiting times and the use of adjuvant chemotherapy, were compared between the short and long survival groups.

The protocol of the present study was in accordance to the ethical guidelines of the 1975 Declaration of Helsinki and was approved by the Institutional Review Committee of Fukushima Medical University School of Medicine (approval no., 2285; Fukushima, Japan). The institutional review board waived the requirement for written informed patient consent due to the retrospective non-interventional nature of the present study.

Statistical analysis. The demographic and clinical characteristic distributions for each group were compared using $\chi^{2}$-tests and Fisher's exact test as appropriate. Variables that followed a Gaussian distribution were assessed using parametric tests, one-way analysis of variance (ANOVA) followed by the post-hoc Tukey's test for differences among all four groups and unpaired Student's t-tests for differences between two groups. Variables that did not follow a Gaussian distribution were log-transformed or assessed using non-parametric tests, Kruskal-Wallis tests (or for non-parametric, ANOVA) for differences among all four groups and Mann-Whitney U-tests for differences between two groups. Survival analysis was 
Table III. Comparisons between patients receiving chemotherapy in short survival and long survival groups ( $\mathrm{n}=72$ ).

\begin{tabular}{|c|c|c|c|}
\hline Characteristics & Short survival $(n=42)$ & Long survival $(n=30)$ & P-value \\
\hline WT1 & $20.0(13.0-28.0)$ & $19.0(11.0-28.0)$ & 0.98 \\
\hline WT2 & $17.0(9.0-30.0)$ & $18.5(12.0-27.0)$ & 0.93 \\
\hline Age (years) & $64.5(58.0-67.0)$ & $66.0(59.0-70.0)$ & 0.31 \\
\hline Tumour size (longest diameter; mm) & $34.0(26.0-45.0)$ & $30.0(23.0-50.0)$ & 0.40 \\
\hline CEA (ng/ml) & $4.1(2.0-13.4)$ & $4.2(2.5-7.5)$ & 0.97 \\
\hline CA19-9 (U/ml) & $1,957.0(116.7-11,025.0)$ & $628.8(99.0-3,197.0)$ & 0.17 \\
\hline \multicolumn{4}{|l|}{$\operatorname{Sex}(n)$} \\
\hline Male & 23 & 16 & 1.00 \\
\hline Female & 19 & 14 & \\
\hline \multicolumn{4}{|l|}{ Tumour location (n) } \\
\hline $\mathrm{Ph}$ & 23 & 14 & 0.63 \\
\hline $\mathrm{Pbt}$ & 19 & 16 & \\
\hline \multicolumn{4}{|l|}{ Disease stage (UICC ver.7) (n) (24) } \\
\hline Locally advanced & 8 & 14 & 0.02 \\
\hline Metastatic & 34 & 16 & \\
\hline \multicolumn{4}{|l|}{ Chemotherapy (n) } \\
\hline Monotherapy & 35 & 18 & 0.003 \\
\hline Combination therapy & 7 & 12 & \\
\hline
\end{tabular}

Mann-Whitney U-test was applied for comparison of continuous variables. Fisher's exact test was applied for comparison of categorical variables. WT1, detection-to-diagnosis waiting time; WT2, diagnosis-to-treatment waiting time; CEA, carcinoembryonic antigen; CA19-9, cancer antigen 19-9; $\mathrm{Ph} / \mathrm{b} / \mathrm{t}$, pancreatic head/body/tail; UICC, Union for International Cancer Control classification.

conducted using the Kaplan-Meier method with log-rank tests. All statistical analyses were performed using GraphPad Prism 6.0 (GraphPad Software, Inc., La Jolla, CA, USA). P<0.05 was considered to indicate a statistically significant difference. Data are presented as the median \pm range.

\section{Results}

Patients. During the study period, 451 patients with PDAC were diagnosed using EUS-FNA. The majority of the patients who were not considered suitable for surgical resection were referred to other hospitals for treatment. Consequently, a total of 149 patients, including 72 patients in the chemotherapy group and 77 patients in the surgical resection group, were included in the present study (Table I). Aside from the aforementioned patients, there were 5 patients who were scheduled originally for surgical resection, but it was cancelled since liver metastasis or peritoneal dissemination was indicated during the waiting time until surgical resection or exploratory laparotomy. WT1 and WT2 of the aforementioned patients, whose surgical resection was cancelled, were 20.0 days (15.0-30.0) and 43.0 days (30.0-55.0), respectively. There were no significant differences in the lengths of WT1 and WT2 between the 79 patients with surgical resection and the 5 patients with the originally planned surgical resection cancelled $(\mathrm{P}=0.70$ and $\mathrm{P}=0.75$, respectively) (Table II).

Survival analysis of patients based on waiting times. The 72 patients who underwent chemotherapy (median OS,
9.7 months; Fig. 1A) were divided into the long survival group $(n=42)$ and the short survival group $(n=30)$ (Table III). No significant differences between the long and short survival groups were identified with respect to WT1 (20.0 vs. 19.0 days; $\mathrm{P}=0.98$ ) or $\mathrm{WT} 2$ (17.0 vs. 18.5 days; $\mathrm{P}=0.93$ ); however, the proportion of patients with locally advanced disease $(\mathrm{P}=0.02)$ and the administration of combination chemotherapy $(\mathrm{P}=0.003)$ were significantly associated with long-term survival, compared with short-term survival (Table III). Among the 77 patients who underwent surgical resection, the median DFS following pancreatic resection was 16.4 months, and the median OS was 35.0 months (Fig. 1B and C). The patients were further divided into short $(n=47)$ vs. long $(n=30)$ DFS groups, and short $(n=57)$ vs. long $(n=20)$ OS groups. Additionally, no significant between-group differences were observed for WT1 in the short vs. long DFS group (21.0 vs. 21.0 days; $\mathrm{P}=0.65)$ and the short vs. long OS group (21.0 vs. 23.0 days; $\mathrm{P}=0.45)$ or WT2 in the short vs. long DFS group (46.0 vs. 44.5 days; $\mathrm{P}=0.45$ ) and the short vs. long OS group (46.0 vs. 41.0 days; $\mathrm{P}=0.36$ ) (Table III). Advanced $\mathrm{T}$ and $\mathrm{N}$ stages were significantly associated with short DFS $(\mathrm{P}=0.049$ and $\mathrm{P}=0.03$, respectively), compared with long DFS; however, only advanced $\mathrm{T}$ stage was significantly associated with short OS ( $\mathrm{P}=0.02$; Table IV).

As indicated in Fig. 2, survival analysis for DFS and OS did not reveal any significant survival differences between patients in the upper and lower quartiles for WT1 or WT2 (Fig. 2A-F). 
Table IV. Comparisons between surgically-treated patients in the short and long survival groups $(\mathrm{n}=77)$.

\begin{tabular}{|c|c|c|c|c|c|c|}
\hline Characteristics & Short DFS (n=48) & Long DFS (n=29) & P-value & Short OS $(n=57)$ & Long OS $(n=20)$ & P-value \\
\hline WT1 (days) & $21.0(15.0-38.0)$ & $21.0(6.0-100.0)$ & 0.65 & $21.0(14.0-36.0)$ & $23.0(17.0-40.0)$ & 0.45 \\
\hline WT2 (days) & $46.0(29.0-62.0)$ & $44.5(13.0-33.7)$ & 0.45 & $46.0(29.0-62.0)$ & $41.0(28.0-55.0)$ & 0.36 \\
\hline Age (years) & $69.0(62.0-77.0)$ & $73.0(69.0-76.0)$ & 0.21 & $69.0(62.0-77.0)$ & $73.0(68.0-75.0)$ & 0.31 \\
\hline $\begin{array}{l}\text { Tumour size } \\
\text { (longest diameter; } \\
\mathrm{mm} \text { ) }\end{array}$ & $19.0(15.0-25.0)$ & $20.0(15.0-20.0)$ & 0.69 & $34.0(8-45.0)$ & $30.0(10.0-30.0)$ & 0.40 \\
\hline CEA (ng/ml) & $3.0(1.9-6.7)$ & $2.45(1.7-3.8)$ & 0.78 & $2.9(1.0-6.2)$ & $2.4(1.8-5.6)$ & 0.97 \\
\hline CA19-9 (U/ml) & $174.6(28.4-518.0)$ & $73.2(16.0-263.0)$ & 0.53 & $109.0(22.5-447.0)$ & $79.7(26.7-270.0)$ & 0.17 \\
\hline \multicolumn{7}{|l|}{ Sex } \\
\hline Male & 29 & 15 & 0.48 & 33 & 11 & 1.00 \\
\hline Female & 19 & 14 & & 24 & 9 & \\
\hline \multicolumn{7}{|l|}{ Tumour location } \\
\hline $\mathrm{Ph}$ & 35 & 17 & 0.21 & 31 & 11 & 1.00 \\
\hline $\mathrm{Pbt}$ & 13 & 12 & & 26 & 9 & \\
\hline \multicolumn{7}{|l|}{$\begin{array}{l}\text { T stage (UICC } \\
\text { ver.7) (24) }\end{array}$} \\
\hline $\mathrm{T} 0-1$ & 4 & 6 & 0.049 & 8 & 2 & 0.02 \\
\hline $\mathrm{T} 2$ & 5 & 7 & & 5 & 7 & \\
\hline T3-4 & 39 & 16 & & 44 & 11 & \\
\hline \multicolumn{7}{|l|}{$\begin{array}{l}\text { N stage (UICC } \\
\text { ver.7) (24) }\end{array}$} \\
\hline N0 & 26 & 23 & 0.03 & 38 & 14 & 1.00 \\
\hline N1 & 22 & 6 & & 19 & 6 & \\
\hline \multicolumn{7}{|l|}{$\begin{array}{l}\text { Adjuvant } \\
\text { chemotherapy }\end{array}$} \\
\hline Yes & 30 & 18 & 1.00 & 36 & 12 & 0.79 \\
\hline No & 18 & 11 & & 21 & 8 & \\
\hline
\end{tabular}

Mann-Whitney U-test was applied for comparison of continuous variables. Fisher's exact test was applied for comparison of categorical variables. WT1, detection-to-diagnosis waiting time; WT2, diagnosis-to-treatment waiting time; CEA, carcinoembryonic antigen; CA19-9, cancer antigen 19-9; Ph/b/t, pancreatic head/body/tail. T, tumor; N, node; DFS, disease-free survival; OS, overall survival; UICC, Union for International Cancer Control classification.

\section{Discussion}

The present retrospective study was conducted in order to elucidate whether prolonged waiting times are associated with the prognosis of unresectable and resectable PDAC. To the best of our knowledge, no prior studies investigated the prognostic effect of waiting time-to-diagnosis in patients with PDAC. Contrary to our hypothesis, the results demonstrated that neither diagnostic nor treatment waiting times significantly affected OS. Additionally, the survival analysis did not reveal any notable differences in survival time between patients who received immediate treatment (patients in the lower quartile of waiting times) and patients whose treatment was delayed (patients in the upper quartile of waiting times).

A total of 3 previous studies that addressed the waiting times for patients with PDAC were identified: Raptis et al (12) combined diagnostic and treatment waiting times and investigated the prognostic impact of overall waiting time. Consistent with the results of the present study, their findings indicated that the length of waiting time from initial referral to diagnosis or treatment did not affect operability, resectability or survival. Jooste et al (11) conducted a population-based study that included 450 patients from two French population-based cancer registries. Factors associated with patient delay (time between the first onset of symptoms and first consultation) and treatment delay (time between first consultation and treatment) were evaluated. Once the results were adjusted for a number of clinical factors, including symptoms and treatments, it was revealed that neither patient nor treatment delay were associated with patient outcomes. Similarly, Sanjeevi et al (13) demonstrated that a short interval between detection of the disease and surgical resection did not affect OS among patients who underwent successful resection of PDAC; however, it may have been associated with a decreased risk of unresectability. Collectively, the aforementioned results did not clearly establish whether timely medical care improves the prognosis of patients with PDAC. 
A

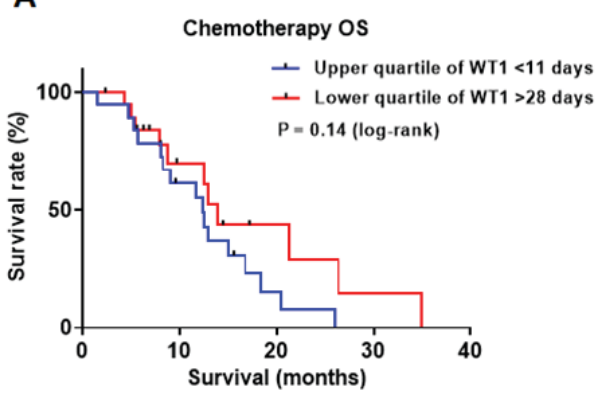

C

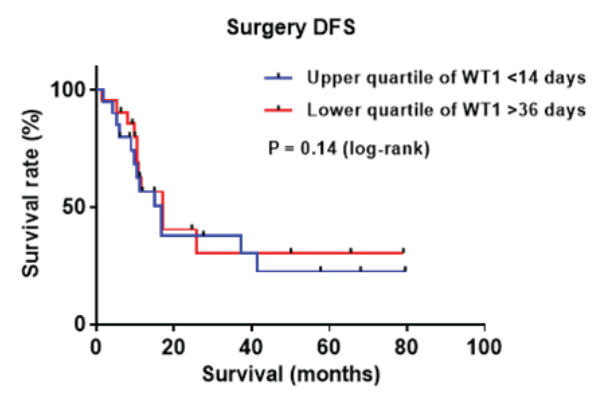

E

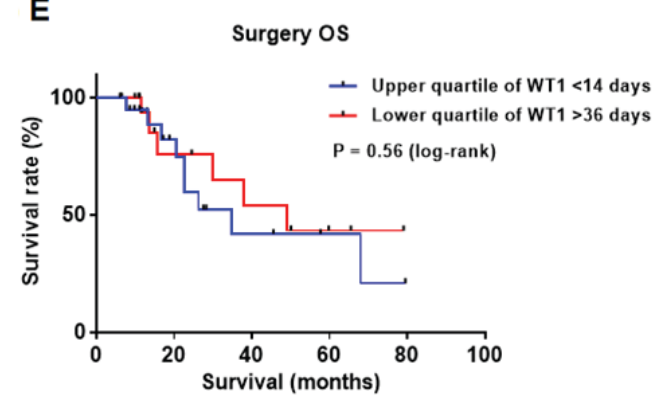

B

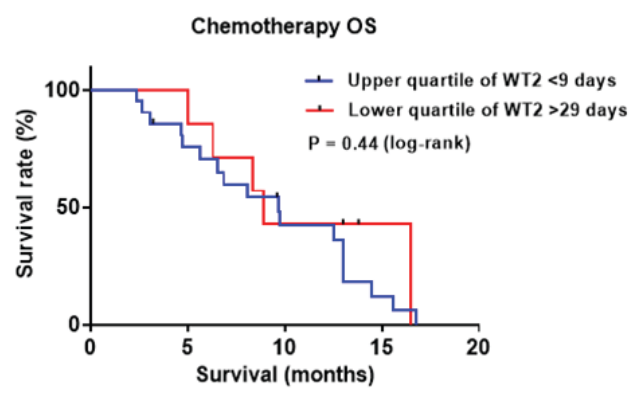

D

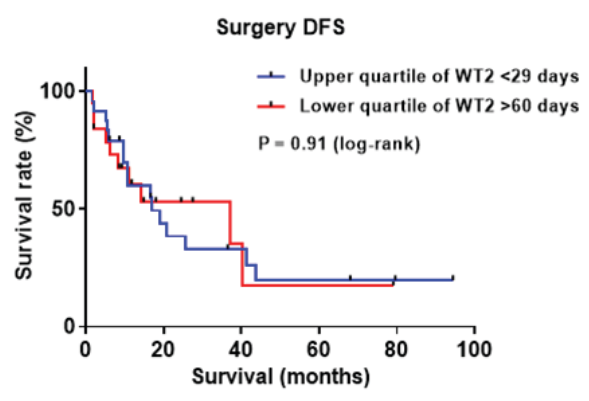

$\mathbf{F}$

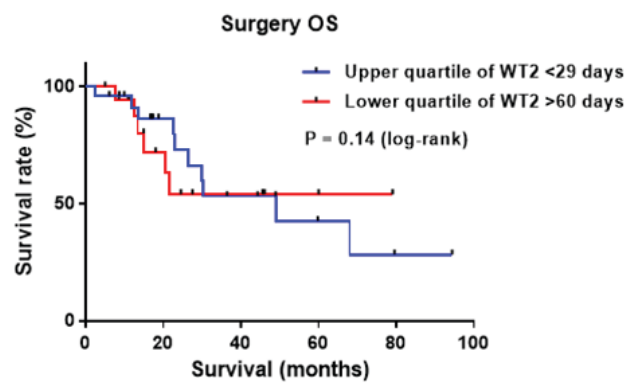

Figure 2. Survival analysis of patients who underwent chemotherapy and surgical resection. Survival analysis (OS) between patients who underwent chemotherapy in the upper and lower quartiles for (A) WT1 and (B) WT2. Survival analysis (DFS) between patients who underwent surgical resection in the upper and lower quartiles for (C) WT1 and (D) WT2. (E and F) Survival analysis (OS) between patients who underwent surgical resection in the upper and lower quartiles for (E) WT1 and (F) WT2. DFS, disease-free survival; OS, overall survival; WT1, detection-to-diagnosis waiting time; WT2, diagnosis-to-treatment waiting time.

The present study was originally conducted based on the hypothesis that prolonged waiting times from detection of disease to diagnosis or to the initiation of treatment may negatively affect patient prognosis. If that hypothesis had been validated, the next step would have been to establish rapid clinical processing of patients to facilitate prompt diagnosis and treatment. However, the results of the present study did not support this hypothesis; nevertheless, healthcare teams should aim to provide medical care to all patients in a timely manner. The results also demonstrated that in accordance with common knowledge, early-stage disease at the initiation of treatment, and the choice of treatment (combination chemotherapy) were significantly associated with good outcomes in PDAC $(3,6,7)$.

However, the present study did have certain limitations, including the fact that it was conducted in a single center with a limited number of patients. Additionally, there may have been selection bias, particularly in patients who were not referred for surgical resection, as the majority of these patients were not treated in Fukushima Medical University Hospital. Furthermore, there could be a possibility that the length of waiting time may affect the choice of treatment, which may subsequently influence prognosis. In fact, 5 patients had their planned surgical resection cancelled due to metastasis detected during waiting time or exploratory laparotomy. However, in terms of the length of waiting time of these patients, the delay in management was not considered a direct cause of this consequence. Patients could have occult metastasis at the time of diagnosis. Additionally, the patients who underwent chemotherapy in Fukushima Medical University Hospital may have had additional reasoning, including comorbidities or good performance status. However, the present study included almost all the surgical candidates who met the inclusion criteria. Therefore, the results of the present study should be validated by assessing an increased number of patients from multiple clinical sites. In conclusion, the present study did not provide any evidence that waiting time is associated with the prognosis in patients with PDAC. 


\section{Acknowledgements}

The abstract was published as abstract no. e15726 in J Clin Oncol 35 (Suppl): 2017.

\section{Funding}

No funding was received.

\section{Availability of data and materials}

All data generated or analyzed during the present study were included in this published article.

\section{Authors' contribution}

RS designed the experiment. RS, TT, TH, MS, NK, HA, KW, JN, SM and HO performed the experiments. RS wrote the manuscript. RS, TT and HA analyzed the data.

\section{Ethics approval and consent to participate}

The present study was approved by the Institutional Review Committee of Fukushima Medical University School of Medicine (approval no., 2285; Fukushima, Japan). The institutional review board waived the requirement for written informed patient consent, due to the retrospective non-interventional nature of the present study.

\section{Patients consent for publication}

Not applicable.

\section{Competing interests}

The authors declare that they have no competing interests.

\section{References}

1. Siegel RL, Miller KD and Jemal A: Cancer statistics, 2016. CA Cancer J Clin 66: 7-30, 2016.

2. Geer RJ and Brennan MF: Prognostic indicators for survival after resection of pancreatic adenocarcinoma. Am J Surg 165: 68-72, 1995.

3. Oettle H, Post S, Neuhaus P, Gellert K, Langrehr J, Ridwelski K, Schramm H, Fahlke J, Zuelke C, Burkart C, et al: Adjuvant chemotherapy with gemcitabine vs observation in patients undergoing curative-intent resection of pancreatic cancer: A randomized controlled trial. JAMA 297: 267-277, 2007.

4. Kang MJ, Jang JY, Chang YR, Kwon W, Jung W and Kim SW: Revisiting the concept of lymph node metastases of pancreatic head cancer: Number of metastatic lymph nodes and lymph node ratio according to N stage. Ann Surg Oncol 21: 1545-1551, 2014

5. Picozzi VJ, Oh SY, Edwards A, Mandelson MT, Dorer R Rocha FG, Alseidi A, Biehl T, Traverso LW, Helton WS and Kozarek RA: Five-year actual overall survival in resected pancreatic cancer: A contemporary single-institution experience from a multidisciplinary perspective. Ann Surg Oncol 24: 1722-1730, 2017.

6. Conroy T, Desseigne F, Ychou M, Bouché O, Guimbaud R, Bécouarn Y, Adenis A, Raoul JL, Gourgou-Bourgade S, de la Fouchardière $C$, et al: FOLFIRINOX versus gemcitabine for metastatic pancreatic cancer. N Engl J Med 364: 1817-1825, 2011.
7. Von Hoff DD, Ervin T, Arena FP, Chiorean EG, Infante J, Moore M, Seay T, Tjulandin SA, Ma WW, Saleh MN, et al: Increased survival in pancreatic cancer with nab-paclitaxel plus gemcitabine. N Engl J Med 369: 1691-1703, 2013.

8. Okusaka T, Ikeda M, Fukutomi A, Ioka T, Furuse J, Ohkawa S, Isayama $\mathrm{H}$ and Boku N: Phase II study of FOLFIRINOX for chemotherapy-naive Japanese patients with metastatic pancreatic cancer. Cancer Sci 105: 1321-1326, 2014.

9. Ueno H, Ioka T, Ikeda M, Ohkawa S, Yanagimoto H, Boku N, Fukutomi A, Sugimori K, Baba H, Yamao K, et al: Randomized phase III study of gemcitabine plus S-1, S-1 alone, or gemcitabine alone in patients with locally advanced and metastatic pancreatic cancer in Japan and Taiwan: GEST study. J Clin Oncol 31: 1640-1648, 2013

10. Dedey F, Wu L, Ayettey H, Sanuade OA, Akingbola TS, Hewlett SA, Tayo BO, Cole HV, de-Graft Aikins A, Ogedegbe G and Adanu R: Factors associated with waiting time for breast cancer treatment in a teaching hospital in Ghana. Health Educ Behav 43: 420-427, 2016.

11. Jooste V, Dejardin O, Bouvier V, Arveux P, Maynadie M, Launoy G and Bouvier AM: Pancreatic cancer: Wait times from presentation to treatment and survival in a population-based study. Int J Cancer 139: 1073-1080, 2016.

12. Raptis DA, Fessas C, Belasyse-Smith P and Kurzawinski TR: Clinical presentation and waiting time targets do not affect prognosis in patients with pancreatic cancer. Surgeon 8: 239-246, 2010.

13. Sanjeevi S, Ivanics T, Lundell L, Kartalis N, Andrén-Sandberg A Blomberg J, Del Chiaro M and Ansorge C: Impact of delay between imaging and treatment in patients with potentially curable pancreatic cancer. Br J Surg 103: 267-275, 2016.

14. Song H, Fang F, Valdimarsdóttir U, Lu D, Andersson TM, Hultman C, Ye W, Lundell L, Johansson J, Nilsson M and Lindblad M: Waiting time for cancer treatment and mental health among patients with newly diagnosed esophageal or gastric cancer: A nationwide cohort study. BMC Cancer 17: 2, 2017.

15. Neal RD, Tharmanathan P, France B, Din NU, Cotton S, Fallon-Ferguson J, Hamilton W, Hendry A, Hendry M, Lewis $\mathrm{R}$, et al: Is increased time to diagnosis and treatment in symptomatic cancer associated with poorer outcomes? Systematic review. Br J Cancer 112 (Suppl 1): S92-S107, 2015.

16. Perri T, Issakov G, Ben-Baruch G, Felder S, Beiner ME, Helpman L, Hogen L, Jakobson-Setton A and Korach J: Effect of treatment delay on survival in patients with cervical cancer: A historical cohort study. Int J Gynecol Cancer 24: 1326-1332, 2014.

17. Chen J, Yang R, Lu Y, Xia Y and Zhou H: Diagnostic accuracy of endoscopic ultrasound-guided fine-needle aspiration for solid pancreatic lesion: A systematic review. J Cancer Res Clin Oncol 138: 1433-1441, 2012.

18. Hewitt MJ, McPhail MJ, Possamai L, Dhar A, Vlavianos P and Monahan KJ: EUS-guided FNA for diagnosis of solid pancreatic neoplasms: A meta-analysis. Gastrointest Endosc 75: 319-331, 2012.

19. Hikichi T, Irisawa A, Bhutani MS, Takagi T, Shibukawa G, Yamamoto G, Wakatsuki T, Imamura H, Takahashi Y, Sato A, et al: Endoscopic ultrasound-guided fine-needle aspiration of solid pancreatic masses with rapid on-site cytological evaluation by endosonographers without attendance of cytopathologists. J Gastroenterol 44: 322-328, 2009.

20. Suzuki R, Lee JH, Krishna SG, Ramireddy S, Qiao W, Weston B, Ross WA and Bhutani MS: Repeat endoscopic ultrasound-guided fine needle aspiration for solid pancreatic lesions at a tertiary referral center will alter the initial inconclusive result. J Gastrointestin Liver Dis 22: 183-187, 2013.

21. Hébert-Magee S, Bae S, Varadarajulu S, Ramesh J, Frost AR, Eloubeidi MA and Eltoum IA: The presence of a cytopathologist increases the diagnostic accuracy of endoscopic ultrasound-guided fine needle aspiration cytology for pancreatic adenocarcinoma: A meta-analysis. Cytopathology 24: 159-171, 2013.

22. Eastern Cooperative Oncology Group. ECOG performance status. http://ecog-acrin.org/resources/ecog-performance-status. Acessed June 3, 2015.

23. International Union against Cancer; Sobin LH, Gospodrowicz MK, Wittekind CH (Eds.), TNM Classification of Malignant Tumours, 7th Ed. New York, NY: Wiley-Liss, 2009. 